\title{
Controlling Energy Transfer in Silicon Quantum Dot Assemblies Made from All-Inorganic Colloidal Silicon Quantum Dots
}

Hiroshi Sugimoto, Kenta Furuta, Minoru Fujii

Department of Electrical and Electronic Engineering, Graduate School of Engineering, Kobe

University, Rokkodai, Nada, Kobe 657-8501, Japan

\section{Estimation of thickness by ellipsometry}

The thicknesses of LbL assemblies of Si QDs are measured by spectroscopic ellipsometer (Auto-SE, Horiba) in the wavelength range of 450 to $1000 \mathrm{~nm}$. The sample is deposited on APTES modified Si wafers with native oxides. The thickness of APTES and native oxides are 2.7 and $0.9 \mathrm{~nm}$, respectively. We assume that the dielectric medium consisting of a Si QD layer $\left(\varepsilon_{e f f}\right)$ is obtained by the Bruggeman effective medium approximation, ${ }^{1}$

$$
f_{S i} \frac{\varepsilon_{S i}-\varepsilon_{e f f}}{\varepsilon_{S i}+2 \varepsilon_{e f f}}+\left(1-f_{S i}\right) \frac{\varepsilon_{a i r}-\varepsilon_{e f f}}{\varepsilon_{a i r}+2 \varepsilon_{e f f}}=0
$$

where $\varepsilon_{S i}$ and $f_{S i}$ are the dielectric permittivity and filling factor of Si and $\varepsilon_{\text {air }}$ is the dielectric permittivity of air. Figure S3 shows the thickness of D-(3.0) $)_{N}$ with filling factor range of 0.25 to 0.35 . 


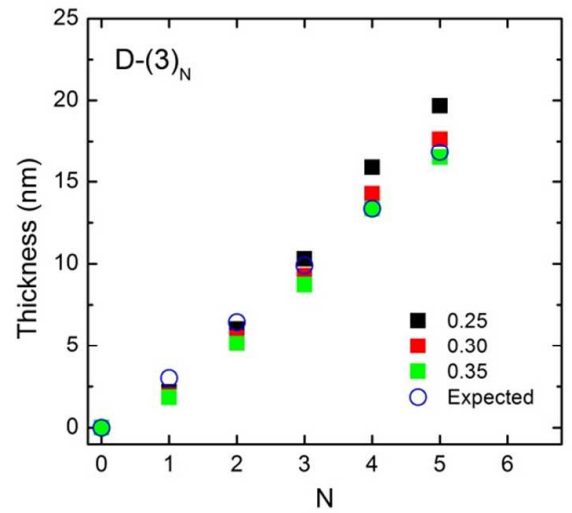

Figure S1. (Filled squares) Thicknesses of D-(3.0) $)_{N}$ obtained by ellipsometry with the filling factor range of 0.25 to 0.35 The data shown as open circles represent the expected thickness of LbL layers of $3 \mathrm{~nm}$ Si QDs and $0.45 \mathrm{~nm}$ PDDA layers. 

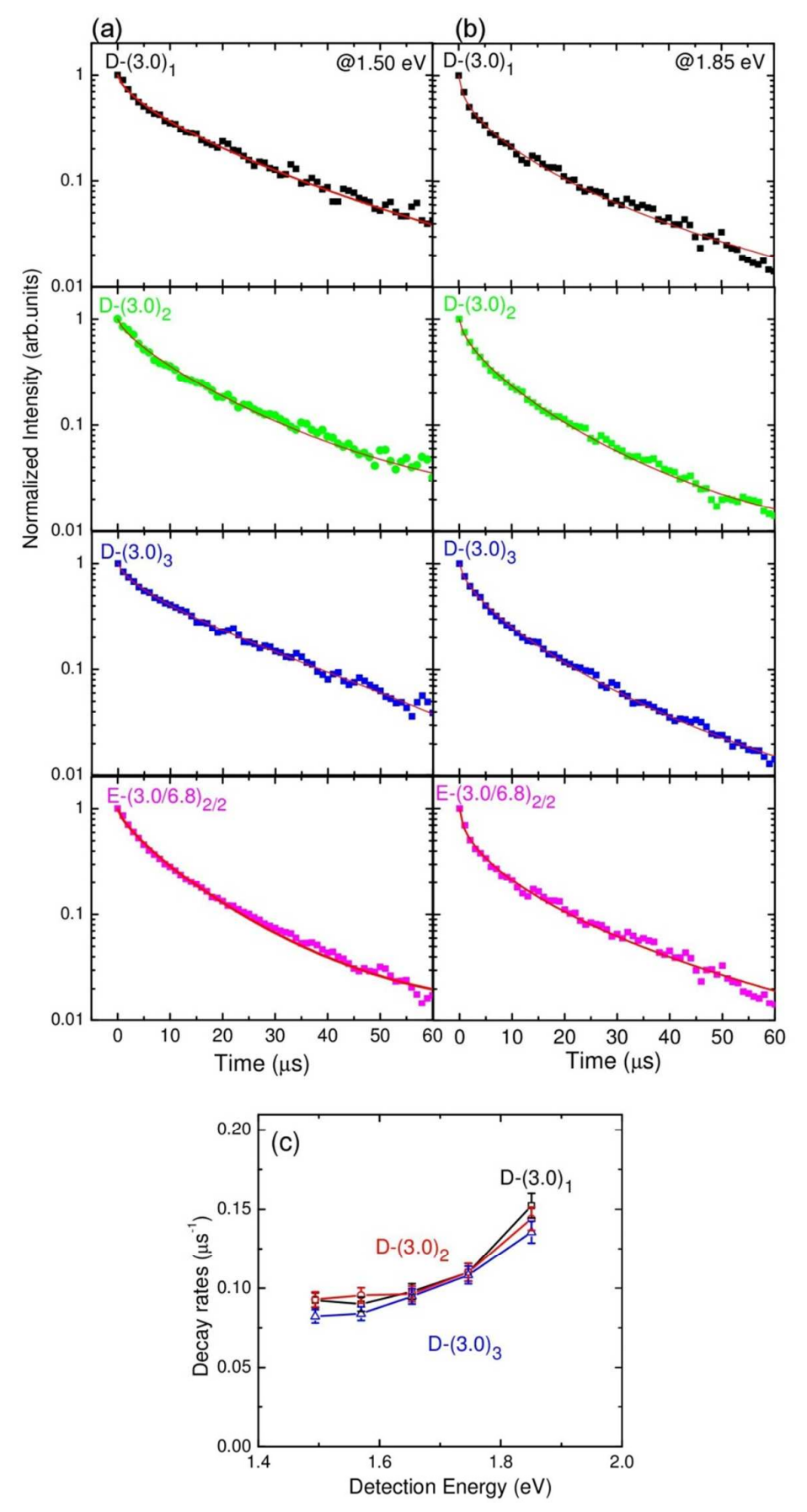

Figure S2. PL decay curves detected at (a) 1.5 and (b) $1.85 \mathrm{eV}$ of D-(3.0) $)_{1}$, D-(3.0) $)_{2}$, $-(3.0)_{3}$ and E-(3.0/6.8) $)_{2 / 2}$ with corresponding stretched exponential fitting results. (c) PL decay rates of D-(3.0) $)_{1}, \mathrm{D}-(3.0)_{2}, \mathrm{D}-(3.0)_{3}$ as a function of detection energy. 


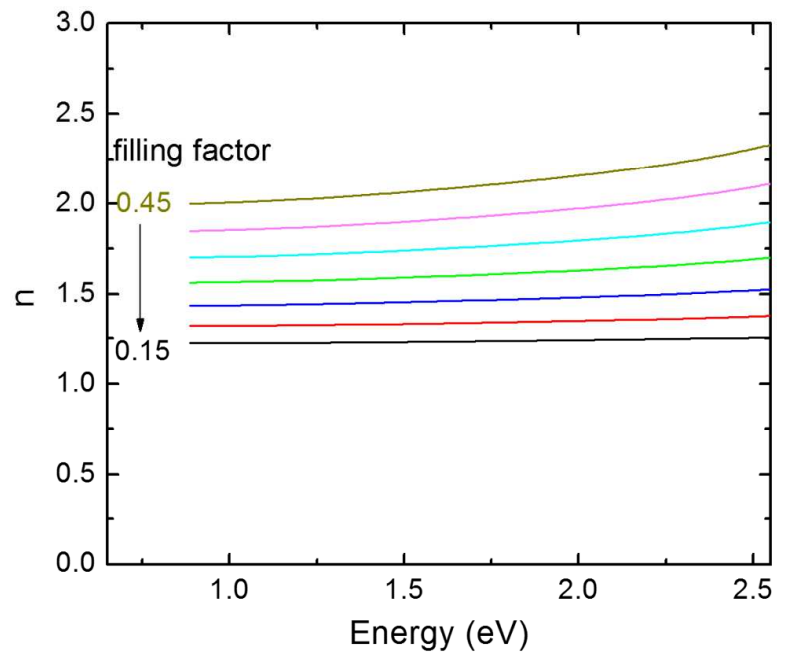

Figure S3. The refractive index as a function of photon energy obtained by the Bruggeman effective medium model with different filling factors of Si QDs.

\section{Reference}

(1) Bruggeman, D. A. G. Calculation of Various Physics Constants in Heterogenous Substances I Dielectricity Constants and Conductivity of Mixed Bodies from Isotropic Substances. Ann. Phys. 1935, 24, 636- 664. 